\title{
Phase space flows for non-Hamiltonian systems with constraints
}

\author{
Alessandro Sergi * \\ Dipartimento di Fisica, Sezione Fisica Teorica, Universitá degli Studi di Messina, \\ Contrada Papardo C.P. 50-98166 Messina, Italy
}

\begin{abstract}
In this paper, non-Hamiltonian systems with holonomic constraints are treated by a generalization of Dirac's formalism. Non-Hamiltonian phase space flows can be described by generalized antisymmetric brackets or by general Liouville operators which cannot be derived from brackets. Both situations are treated. In the first case, a Nosé-Dirac bracket is introduced as an example. In the second one, Dirac's recipe for projecting out constrained variables from time translation operators is generalized and then applied to non-Hamiltonian linear response. Dirac's formalism avoids spurious terms in the response function of constrained systems. However, corrections coming from phase space measure must be considered for general perturbations.
\end{abstract}

\section{INTRODUCTION}

Constrained systems are ubiquitous in theory and computation and formalisms for their treatment are still developed 1, 2, 3]. Some time ago Dirac showed how to formulate generalized Hamiltonian phase space flows which automatically satisfy certain class of constraints [4, 5, 6, 7, 8]. These constraints, which are called second class, are specified by a non-zero Poisson bracket with the Hamiltonian. Dirac's investigation aimed at finding a quantization procedure for relativistic fields which have constraints arising from their Lorentz or gauge symmetries. Recently it has been shown [3] how Dirac's scheme can be also applied to non-relativistic systems, such as those addressed by classical molecular dynamics simulations in condensed matter, where constraints are used to describe the topology of molecules or rare events [9]. In particular it has been shown how Dirac's formalism can be subsumed by means of a generalized bracket introduced in Refs. [10, 11].

The concern of this paper is to generalize the approach of 3 to cases where the dynamics is non-Hamiltonian 10 , $11,12,13,14,15,16,17]$. This is useful since computational schemes adopt constraints and non-Hamiltonian dynamics at the same time, with the latter implementing both specific thermodynamic conditions, i.e. constant temperature [18, 19, 20, 21], and nonequilibrium perturbations not derivable from a Hamiltonian [22, 23, 24, 25]. Building on the results given in Ref. [3], the discussion will be limited to systems with holonomic constraints. Nonholonomic constraints (such as those involved in the formulation of the isokinetic ensemble 1, 2] ) will not be addressed in this paper. Two kinds of non-Hamiltonian dynamics will be considered. The first is based on the generalized brackets introduced in [10, 11] while the second case arises from Liouville operators which cannot be expressed by means of antisymmetric brackets. For the first case, the antisymmetric structure of the generalized bracket will be used to combine Dirac's the-

*(E-mail: asergi@unime.it) ory with non-Hamiltonian Nosé-Hoover dynamics (more general dynamics, such as Nosé-Hoover chains [20], do not introduce any conceptual difference). As an application of the Nosé-Dirac phase space flow, the unbiasing factor, arising when holonomic constraints are used to study rare events [9], is re-derived. The second type of non-Hamiltonian dynamics requires a generalization of Dirac's scheme in order to project out the constrained degrees of freedom from any arbitraty Liouville operator. Linear response theory will be reviewed and some fine points, which are relevant for analyzing dynamics with holonomic constraints (such as in the case of molecular systems [26, 27, 28, 29]), will be discussed. In particular, it will be shown that correction terms, stemming from phase space measure, appear in the response function for general forms of perturbations.

The paper is organized as follows. In Sec. II Dirac's Hamiltonian formalism is briefly reviewed. In Sec. III a unified bracket for Nosé thermostated dynamics and constraints, producing a non-Hamiltonian Nosé-Dirac phase space flow, is introduced. As an illustration of the formalism, the Nosé-Dirac flow is applied in Appendix A to the discussion of rare events sampling. In Sec. IV] Dirac's recipe, for projecting out the spurious dynamics of constrained variables, is first generalized to arbitrary time-translation operators and then applied to non-Hamiltonian Liouville operators which cannot be derived from brackets. Linear response theory is briefly reviewed, discussing how Dirac's prescription avoids fake terms coming from constraints. Nevertheless, it is shown that corrections terms in the response function, originating from the constrained phase space measure, may appear in the general case.

\section{HAMILTONIAN FORMALISM FOR SYSTEMS WITH HOLONOMIC CONSTRAINTS}

Consider a system with a conserved energy $\mathcal{H}_{0}(\mathbf{x})$, where $\mathbf{x}=(\mathbf{r}, \mathbf{p})$ denotes the phase space point. To formulate phase space equations of motion in the presence of mechanical constraints one can follow Dirac's approach $[3,4,5,6,7,8]$. Together with the $n$ constraints in 
configuration space $\boldsymbol{\sigma}(\mathbf{r})=0$, one has to consider an additional number $n$ of phase space constraints $\dot{\boldsymbol{\sigma}}(\mathbf{r}, \dot{\mathbf{r}})=0$. It is useful to let $\chi=(\boldsymbol{\sigma}, \dot{\boldsymbol{\sigma}})$ denote the entire set of $2 n$ phase space constraints. The $n$ constraints $\dot{\boldsymbol{\sigma}}=0$ are redundant but necessary to set up a phase space picture of the dynamics. Following the convention (due to Dirac [4, 5]) of evaluating derivatives first and imposing constraint relations after, these constraints will disappear from the equations of motion and will not contribute to the phase space measure.

The equations of motion with constraints may be written as [3]

$$
\dot{x}_{i}=\sum_{j=1}^{2 N} \mathcal{B}_{i j}^{D}(\mathbf{x}) \frac{\partial \mathcal{H}_{0}}{\partial x_{j}}
$$

where $2 N$ is phase space dimension and $\mathcal{B}^{D}$ is an antisymmetric tensor defined by

$$
\mathcal{B}_{i j}^{D}(x)=\mathcal{B}_{i j}^{c}-\sum_{k, l}^{2 N} \sum_{\alpha, \beta}^{2 n} \mathcal{B}_{i k}^{c} \frac{\partial \chi_{\alpha}}{\partial x_{k}}\left(\mathbf{C}^{-1}\right)_{\alpha \beta} \frac{\partial \chi_{\beta}}{\partial x_{l}} \mathcal{B}_{l j}^{c},
$$

with

$$
\mathcal{B}^{c}=\left[\begin{array}{cc}
0 & 1 \\
-1 & 0
\end{array}\right]
$$

usually called the symplectic matrix [30]. In order to arrive at Eq. (2) one has to define

$$
C_{\alpha \beta}=\left\{\chi_{\alpha}, \chi_{\beta}\right\}=\sum_{i j} \frac{\partial \chi_{\alpha}}{\partial x_{i}} \mathcal{B}_{i j}^{c} \frac{\partial \chi_{\beta}}{\partial x_{j}}
$$

given in terms of Poisson bracket of the $2 n$ phase space constraints, and the inverse matrix $\left(\mathbf{C}^{-1}\right)_{\alpha \beta}(\alpha, \beta=$ $1, \ldots, 2 n)$,written explicitly in block form as

$$
\mathbf{C}^{-1}=\left[\begin{array}{cc}
\boldsymbol{Z}^{-1} \boldsymbol{\Gamma} \boldsymbol{Z}^{-1} & -\boldsymbol{Z}^{-1} \\
\boldsymbol{Z}^{-1} & \mathbf{0}
\end{array}\right]
$$

where the matrices

$$
Z_{\alpha \beta}=\sum_{i=1}^{N} \frac{1}{m_{i}} \nabla_{i} \sigma_{\alpha} \nabla_{i} \sigma_{\beta}
$$

and

$$
\Gamma_{\alpha \beta}=\sum_{i, k=1}^{N} \frac{p_{i}}{m_{i} m_{k}}\left(\nabla_{k i}^{2} \sigma_{\alpha} \nabla_{k} \sigma_{\beta}-\nabla_{k} \sigma_{\alpha} \nabla_{k i}^{2} \sigma_{\beta}\right)
$$

(with $\alpha, \beta=1, \ldots, n$ ) have been defined.

The matrix $\mathcal{B}^{D}$ can be written explicitly in block form as

$$
\mathcal{B}^{D}=\left[\begin{array}{cc}
\mathbf{0} & \mathbf{1}-\boldsymbol{\Delta} \\
-\mathbf{1}+\boldsymbol{\Delta}^{T} & \boldsymbol{\Lambda}
\end{array}\right],
$$

where

$$
\begin{aligned}
\Delta_{i j}= & \sum_{\substack{k, l=1 \\
(i, j=1, \ldots, N)}}^{N} \sum_{\alpha, \beta=1}^{n} \mathcal{B}_{i, N+k}^{c} \frac{\partial \dot{\sigma}_{\alpha}}{\partial \mathbf{p}_{k}}\left(\boldsymbol{Z}^{-1}\right)_{\alpha \beta} \frac{\partial \sigma_{\beta}}{\partial \mathbf{r}_{l}} \mathcal{B}_{l, N+j}^{c} \\
& (i, j=1, \ldots, N)
\end{aligned}
$$

and

$$
\begin{aligned}
\Lambda_{i j}= & -\sum_{\substack{k, l=1 \\
(i, j=1, \ldots, N=1}}^{N} \sum_{\alpha, N)}^{n} \mathcal{B}_{N+i, k}^{c} \frac{\partial \sigma_{\alpha}}{\partial \mathbf{r}_{k}}\left(\boldsymbol{Z}^{-1} \boldsymbol{\Gamma} \boldsymbol{Z}^{-1}\right)_{\alpha \beta} \frac{\partial \sigma_{\beta}}{\partial \mathbf{r}_{l}} \mathcal{B}_{l, N+j}^{c} \\
& (10)
\end{aligned}
$$

Substituting $\mathcal{B}^{D}$ into Eq. (1) and taking into account the fact that $\dot{\boldsymbol{\sigma}}=0$, one obtains the equations of motion [3]

$$
\begin{aligned}
\dot{\mathbf{r}}_{i} & =\frac{\mathbf{p}_{i}}{m_{i}} \\
\dot{\mathbf{p}}_{i} & =\mathbf{F}_{i}+\nabla_{i} \boldsymbol{\sigma} \cdot \boldsymbol{\lambda}(\mathbf{r}, \mathbf{p})
\end{aligned}
$$

where

$$
\boldsymbol{\lambda}(\mathbf{r}, \mathbf{p})=\boldsymbol{Z}^{-1} \cdot\left\{\dot{\boldsymbol{\sigma}}, \mathcal{H}_{0}\right\}
$$

Equations (11)-(12) have a phase space compressibility 3,31$]$

$$
\kappa_{c}=-\frac{d}{d t} \ln \|\boldsymbol{Z}\|
$$

and distribution function $[3,14$

$$
\rho_{e}=\delta\left(\mathcal{H}_{0}\right) \delta(\boldsymbol{\chi})\|\boldsymbol{Z}\|
$$

where $\delta(\boldsymbol{\chi})=\prod_{\alpha} \delta\left(\boldsymbol{\sigma}_{\alpha}\right) \delta\left(\boldsymbol{\sigma}_{\alpha}\right)$. It is worth to remark that Eqs. (1) with the tensor in Eq. (2), and their explicit form (11)-(12), can be regarded as Hamiltonian since the associated generalized bracket

$$
(a, b)_{D}=\sum_{i, j=1}^{N} \frac{\partial a}{\partial x_{i}} \mathcal{B}_{i j}^{D} \frac{\partial b}{\partial x_{j}}
$$

where $a$ and $b$ are arbitrary phase space functions, satisfies the Jacobi relation [3, 32]. The generalized bracket in Eq. (16) has the property of leaving invariant, by construction, any function of the constraints.

\section{NOSÉ-DIRAC PHASE SPACE FLOW}

Starting from the structure of either (11) or the associated generalized bracket in (16), with $\mathcal{B}^{D}$ given by Eq. (2), it is very easy to define non-Hamiltonian equations of motion. It suffices to substitute the tensor $\boldsymbol{B}^{c}$ in Eq. (2) with a more general antisymmetric tensor $\mathcal{B}(x)$ so that the Jacobi identity is no longer satisfied. When one tries to apply this program to extended system dynamics, as in the case of Nosé-Hoover dynamics, problems 
are encountered since the constraints are usually defined only onto a subspace of the extended phase space variables. This straightforward approach would make the generalized bracket identically zero. One can bypass this problem by exploiting the block structure of $\mathcal{B}^{D}$ as given in (8). To this end, consider Nosé extended phase space with coordinates $\mathbf{x}=\left(\mathbf{r}, \eta, \mathbf{p}, p_{\eta}\right)$, and introduce the Nosé Hamiltonian

$$
\begin{aligned}
\mathcal{H}_{\mathrm{N}} & =\sum_{i}^{N} \frac{\mathbf{p}_{i}^{2}}{2 m_{i}}+\Phi(\mathbf{r})+\frac{p_{\eta}^{2}}{2 m_{\eta}}+g k_{B} T \eta \\
& =\mathcal{H}_{\mathrm{T}}+g k_{B} T \eta .
\end{aligned}
$$

If one defines the antisymmetric matrix

$$
\mathcal{B}=\left[\begin{array}{cccc}
0 & 0 & 1-\Delta & 0 \\
0 & 0 & 0 & 1 \\
-1+\Delta & 0 & \Lambda & -p \\
0 & -1 & p & 0
\end{array}\right]
$$

and uses this matrix in place of $\mathcal{B}^{D}$ either in (11) or in (16), then, through the Nosé Hamiltonian in Eq. (18), one obtains the desired equations of motion,

$$
\begin{aligned}
\dot{\mathbf{r}}_{i} & =\frac{\mathbf{p}}{m_{i}} \\
\dot{\mathbf{p}}_{i} & =\mathbf{F}_{i}+\nabla \boldsymbol{\sigma} \cdot \boldsymbol{\lambda}(\mathbf{r}, \mathbf{p})-\mathbf{p}_{i} \frac{p_{\eta}}{m_{\eta}} \\
\dot{\eta} & =\frac{p_{\eta}}{m_{\eta}} \\
\dot{p}_{\eta} & =F_{\eta},
\end{aligned}
$$

with $F_{\eta}=\sum_{i} \mathbf{p}_{i}^{2} / m_{i}-g k_{B} T$.

The phase space flow defined via $\mathcal{B}$ in Eq. (19) conserves the Hamiltonian and any function of the constraints. The equations of motion (Eqs. (20)-(23)) have a compressibility

$$
\kappa=\sum_{i j}^{2 N} \frac{\partial \mathcal{B}_{i j}}{\partial x_{i}} \frac{\partial \mathcal{H}}{\partial x_{j}}=\kappa_{c}+\kappa_{N}
$$

where $\kappa_{c}$ is given in Eq. (14). The Nosé compressibility is $\kappa_{N}=3 N \dot{\eta}$ so that the total compressibility of Eqs (20)(23) is

$$
\kappa=-\frac{d \ln |\boldsymbol{Z}|}{d t}+\beta \frac{d \mathcal{H}_{T}}{d t} .
$$

The primitive function of the compressibility is $w(x)=$ $-\ln |\boldsymbol{Z}|+\beta \mathcal{H}_{T}$ so that the distribution function in the extended phase space is

$$
\rho_{\mathrm{ND}}\left(\mathbf{r}, \mathbf{p}, \eta, p_{\eta}\right)=\delta\left(\mathcal{H}_{\mathrm{N}}\right) \delta(\boldsymbol{\sigma}) \delta(\dot{\boldsymbol{\sigma}})|\boldsymbol{Z}| e^{-\beta \mathcal{H}_{T}} .
$$

One can easily prove that Eq. (26) provides the distribution of a canonical ensemble with constraints. Integrating on $\eta$ one has

$$
\int d \eta \delta\left(\mathcal{H}_{\mathrm{N}}(\eta)\right)=\int d \eta \delta(\eta)\left[\frac{d \mathcal{H}_{\mathrm{N}}}{d \eta}\right]^{-1}=\frac{\beta}{g}
$$

The constant can be absorbed in the normalization and the Gaussian integration on $p_{\eta}$ can be easily performed so that one obtains

$$
\rho_{c}(\mathbf{r}, \mathbf{p})=\delta(\boldsymbol{\chi})|\boldsymbol{Z}| e^{-\beta \mathcal{H}_{0}},
$$

where $\mathcal{H}_{0}=\sum_{i} \mathbf{p}_{i}^{2} / 2 m_{i}+\Phi(\mathbf{r})$ is the Hamiltonian of the physical degrees of freedom. As an example, Nosé-Dirac flow will be applied in Appendix A to the sampling of rare events, and it will be shown how to re-derive the unbiasing factor first introduced in Ref. 9].

\section{GENERAL NON-HAMILTONIAN DYNAMICS AND CONSTRAINTS}

In the case of general non-Hamiltonian dynamics one cannot derive the generator of time translation from generalized brackets. Instead one is led to consider a Liouville operator [23] of the form

$$
i L_{\mathrm{p}}=\sum_{i=1}^{N} \mathbf{C}(\{\mathbf{r}, \mathbf{p}\}) \cdot \frac{\partial}{\partial \mathbf{r}_{i}}+\mathbf{D}(\{\mathbf{r}, \mathbf{p}\}) \cdot \frac{\partial}{\partial \mathbf{p}_{i}}
$$

defining the time evolution of any arbitrary phase space variable $a(\{\mathbf{r}, \mathbf{p}\})$ through $\dot{a}=i L_{\mathrm{p}} a$. The phase space incompressibility condition is usually adopted [23]

$$
\frac{\partial \mathbf{C}}{\partial \mathbf{r}_{i}}+\frac{\partial \mathbf{D}}{\partial \mathbf{p}_{i}}=0
$$

and for simplicity the same will be done here. In molecular dynamics applications, Liouville operators, having the same form as that in Eq. (29), are used to introduce time dependent perturbations by means of operators of the form

$$
i L_{I}(t)=\mathcal{F}(t) i L_{\mathrm{p}}
$$

The unperturbed systems is usually subjected to the action of an operator $i L_{0}$ which is instead derivable from some (generalized or Poisson) bracket with the Hamiltonian. Accordingly, the total dynamics is defined via the operator $i L(t)=i L_{0}+i L_{I}(t)$. In the presence of holonomic constraints, for example describing rigid molecules, the formalism of Ref. 3], for the Hamiltonian case, or of the previous section, for non-Hamiltonian dynamics, can be used to define an operator $i L_{0}^{D}$ having the constraints as conserved quantities. The problem is that $i L_{\mathrm{p}}$ and $i L_{I}(t)$ as such do not preserve the constraints and could lead to spurious term in the linear response derivation, as it will be shown in the following. This feature of the formalism is not desiderable since, in actual molecular dynamics calculations, the algorithms enforcing the constraints is used in the presence of the perturbation determined by $i L_{I}(t)$ [26, 27, 28, 29] so that this perturbation does not violate the constraints in practice. The conclusion is that, in order to set up a correct formalism, one must project out the dynamics that $i L_{\mathrm{p}}$ 
and $i L_{I}(t)$ spuriously impose on the constraints. To this aim, by using a simple extension of Dirac's recipe to general non-Hamiltonian Liouville operators, one can define $i L_{I}^{D}(t)$ as follows

$$
\begin{aligned}
i L_{I}^{D}(t) a & =i L_{I}(t) a \\
& -\sum_{\alpha \beta}\left\{a, \chi_{\alpha}\right\}\left(\mathbf{C}^{-1}\right)_{\alpha \beta}\left(i L_{I}(t) \chi_{\beta}\right) \\
& =\mathcal{F}(t)\left[i L_{\mathrm{p}} a\right. \\
& \left.-\sum_{\alpha \beta}\left\{a, \chi_{\alpha}\right\}\left(\mathbf{C}^{-1}\right)_{\alpha \beta}\left(i L_{\mathrm{p}} \chi_{\beta}\right)\right] \\
& =\mathcal{F}(t) i L_{\mathrm{p}}^{D} a,
\end{aligned}
$$

where $a$ is an arbitrary phase space variable, $\left\{a, \chi_{\alpha}\right\}$ is the Poisson bracket and $\mathbf{C}$ is derived by means of Eqs. (4) and (5). As in the Hamiltonian case [3] or in that of the non-Hamiltonian bracket, $i L_{I}^{D}(t)$ preserves any function of the constraints, by construction. Equation (32) generalizes Dirac's theory to arbitrary non-Hamiltonian phase space flows.

By means of $i L_{I}^{D}(t)$ one can set up the correct formalism for the linear response of systems with holonomic constraints subject to a non-Hamiltonian time-dependent perturbation. For simplicity, the case in which the unperturbed dynamics of the constrained system is Hamiltonian will be considered in the following. In this situation, the unperturbed system has a conserved Hamiltonian $\mathcal{H}_{0}$, a Liouville operator $i L_{0}^{D}=\sum_{i} \mathcal{B}_{i j}^{D} \partial / \partial x_{i}$, with $\mathcal{B}^{D}$ defined by Eq. (2), and an equilibrium distribution function given by $\rho_{e}=\|\boldsymbol{Z}\| \delta(\boldsymbol{\chi}) \delta\left(\mathcal{H}_{0}\right)$. The Liouville equation in the presence of the perturbation is

$$
\frac{\partial \rho}{\partial t}=-i \mathcal{L}_{0}^{D} \rho-i L_{I}^{D}(t) \rho
$$

where [3] $i \mathcal{L}_{0}^{D}=i L_{0}^{D}+\kappa_{c}$. One can consider $\rho=\rho_{e}+\delta \rho$ and to linear order

$$
\delta \rho(t)=-\int_{0}^{t} d \tau \mathcal{F}(\tau) e^{-i \mathcal{L}_{0}^{D}(t-\tau)} i L_{\mathrm{p}}^{D} \rho_{e} .
$$

The nonequilibrium average of $\delta b(t)=b(t)-\langle b\rangle_{e q}$ for any phase space variable is then

$$
\overline{\delta b}(t)=\int_{0}^{t} d \tau \mathcal{F}(\tau) \phi(t-\tau)
$$

where

$$
\phi(t)=-\int d x b(t) i L_{\mathrm{p}}^{D} \rho_{e}
$$

is the response function and $b(t)=\exp \left[i L_{0}^{D} t\right] b(0)$ since the compressibility $\kappa_{c}$ disappears when integrating by parts $\exp \left[i \mathcal{L}_{0}^{D} t\right][3,11]$. Now, in evaluating the action of $i L_{\mathrm{p}}^{D}$ on $\rho_{e}$ one can take full advantage of the fact that $i L_{\mathrm{p}}^{D} \delta(\chi)=0$. Had one used $i L_{\mathrm{p}}$ instead, spurious terms would have appeared. Thus

$$
i L_{\mathrm{p}}^{D} \rho_{e}=\rho_{e}\left[i L_{\mathrm{p}}^{D} \ln \|\boldsymbol{Z}\|-\beta\left(i L_{\mathrm{p}}^{D} \mathcal{H}_{0}\right)\right],
$$

where it has been used the fact that $i L_{\mathrm{p}}^{D} \delta\left(\mathcal{H}_{0}\right) \approx$ $-\beta \delta\left(\mathcal{H}_{0}\right)\left(i L_{\mathrm{p}}^{D} \mathcal{H}_{0}\right)$ in the thermodynamic limit [33]. Hence, the response function for constrained systems takes the form

$$
\phi(t)=\beta\left\langle\left(i L_{\mathrm{p}}^{D} \mathcal{H}_{0}-\beta^{-1} i L_{\mathrm{p}}^{D} \ln \|\boldsymbol{Z}\|\right)\right\rangle_{e q} .
$$

The correction factor arising from $i L_{\mathrm{p}}^{D} \ln \|\boldsymbol{Z}\|$ disappears if

$$
i L_{\mathrm{p}}=\sum_{i} \mathbf{D}_{i} \partial / \partial \mathbf{p}_{i}
$$

but for general equations of motion [22, 23, 24, 25, 26, 27, 28, 29] it must be considered.

\section{CONCLUSIONS}

The extension of Dirac's formalism allows one to treat correctly systems with holonomic constraints undergoing non-Hamiltonian dynamics. Non-Hamiltonian dynamics can be derived from generalized brackets or it can be more general and not be derivable from any brackets: both cases have been treated. Using generalized brackets, a Nosé-Dirac phase space flow has been introduced and applied to derive the unbiasing factor when constraints are used to sample rare events. It has been shown how to generalize Dirac's recipe when the dynamics cannot be obtained from brackets. Linear response theory of system with holonomic constraints subjected to general non-Hamiltonian perturbation has been illustrated. The use of Dirac's formalism makes spurious terms disappear from the response function. However, a correction coming from the measure of constrained phase space is present in general cases. Further work is required in order to assess the importance of this correction in numerical calculations on condensed matter systems.

Equilibrium statistical mechanics and linear response theory of systems with nonholonomic constraints remain to be addressed. However, as a consequence of the analysis presented in this paper, it can be suggested that a formalism, suitable for the linear response of such systems, must project the spurious time evolution of the constrained variables out of both unperturbed and perturbed dynamics.

\section{Acknowledgments}

The author is grateful to Professor Raymond Kapral for many discussions. 


\section{APPENDIX A: NOSÉ-DIRAC FLOW AND RARE EVENTS}

Often one is interested in the calculation of conditional averages of some phase space function $a(\mathbf{r})$

$$
\langle a(\mathrm{r})\rangle_{\mathrm{cond}}=\frac{\left\langle a(\mathrm{r}) \delta\left(\boldsymbol{\xi}(\mathbf{r})-\boldsymbol{\xi}^{\ddagger}\right)\right\rangle_{N V T}}{\left\langle\delta\left(\boldsymbol{\xi}(\mathbf{r})-\boldsymbol{\xi}^{\ddagger}\right)\right\rangle_{N V T}}
$$

where $\langle\ldots\rangle_{N V T}$ stands for an equilibrium average in the canonical ensemble. Molecular dynamics can be used to perform calculation where the condition $\boldsymbol{\sigma}(\mathbf{r})=\boldsymbol{\xi}-\boldsymbol{\xi}^{\ddagger}=$ 0 is treated as a holonomic constraint. However this automatically brings in a constraints on the time variation $\dot{\boldsymbol{\sigma}}(\mathbf{r}, \dot{\mathbf{r}})$ so that using the Nosé-Dirac flow introduced before one would get a constrained average, defined by

$$
\langle a(\mathbf{r})\rangle_{\xi^{\ddagger}}=\frac{\langle a(\mathbf{r})\|\boldsymbol{Z}\| \delta(\boldsymbol{\chi})\rangle_{N V T}}{\langle\|\boldsymbol{Z}\| \delta(\boldsymbol{\chi})\rangle_{N V T}}
$$

where $\delta(\boldsymbol{\chi})=\delta(\boldsymbol{\sigma}) \delta(\dot{\boldsymbol{\sigma}})$.
The relation between conditional average A1 and constrained averages A2 has been originally given in Ref. 9]. In the present context, it is simply remarked that, since the formal manipulations are performed in the canonical ensemble, in order to be rigorous one needs the Nosé-Dirac flow to have the correct distribution function given in Eq. (28). Having said that, one just needs the results of Ref [34], which show that $\int d^{N} \mathbf{p}\|\boldsymbol{Z}\| \delta(\dot{\boldsymbol{\sigma}}) \propto$ $\|\boldsymbol{Z}\|^{1 / 2}$, in order to re-write the constrained average as

$$
\langle a(\mathbf{r})\rangle_{\xi^{\ddagger}}=\frac{\left\langle a(\mathbf{r})\|\boldsymbol{Z}\|^{1 / 2} \delta(\boldsymbol{\sigma})\right\rangle_{N V T}}{\left\langle\|\boldsymbol{Z}\|^{1 / 2} \delta(\boldsymbol{\sigma})\right\rangle_{N V T}} .
$$

From this, one immediately obtains the result of Ref. 9]

$$
\langle a(\mathbf{r})\rangle_{\text {cond }}=\frac{\left\langle|\boldsymbol{Z}|^{-1 / 2} a(\mathbf{r})\right\rangle_{\xi^{\ddagger}}}{\left\langle|\boldsymbol{Z}|^{-1 / 2}\right\rangle_{\xi^{\ddagger}}} .
$$

[1] P. Minary, G. J. Martyna and M. E. Tuckerman, J. Chem. Phys. 1182510 (2003).

[2] P. Minary, G. J. Martyna and M. E. Tuckerman, J. Chem. Phys. 1182527 (2003).

[3] A. Sergi, Phys. Rev. E 69021109 (2004).

[4] P. A. M. Dirac, Can. J. of Math. 2147 (1950).

[5] P. A. M. Dirac, Lessons in Quantum Mechanics, Dover New York (2001).

[6] E. C. G. Sudarshan and N. Mukunda, Classical Mechanics: A Modern Perspective, Wiley New York (1974).

[7] A. J. Hanson, T. Regge and C. Teitelboim Constrained Hamiltonian Systems, Accademia Nazionale dei Lincei (1976).

[8] K. Sundermeyer, Constrained Dynamics, Lecture notes in Physics Springer-Verlag New York (1982).

[9] E. A. Carter, G. Ciccotti, J. T. Hynes and R. Kapral, Constrained reaction coordinate dynamics for the simulation of rare events, Chem. Phys. Lett. 156:472-477 (1989).

[10] A. Sergi and M. Ferrario, Phys. Rev. E 64056125 (2001).

[11] A. Sergi, Phys. Rev. E 67021101 (2003).

[12] M. E. Tuckerman, C. J. Mundy and M. L. Klein, Phys. Rev. Lett. 782042 (1997).

[13] M. Tuckerman, C. J. Mundy and G. J. Martyna, Europhys. Lett. 45149 (1999).

[14] M. E. Tuckerman, Y. Liu, G. Ciccotti and G. L. Martyna, J. Chem. Phys. 1151678 (2001).

[15] J. D. Ramshaw, Europhys. Lett. 59319 (2002).

[16] G. S. Ezra, J. Math. Chem. 32339 (2002).

[17] G. S. Ezra, J. Math. Chem. 3529 (2004).
[18] S. Nosé, Molec. Phys. 52255 (1984).

[19] W. G. Hoover, Phys. Rev. A 311695 (1985).

[20] G. J. Martyna, M. L. Klein and M. Tuckerman, J. Chem. Phys. 922635 (1992).

[21] M. Ferrario, in Computer simulation in Chemical Physics Kluwer Academy (1993).

[22] A. J. C. Ladd, Mol. Phys. 53459 (1984).

[23] D. J. Evans and G. P. Morriss, Comp. Phys. Rep. 1297 (1984).

[24] D. J. Evans and G. P. Morriss, Statistical Mechanics of Nonequilibrium Liquids, Academic, New York (1990).

[25] M. E. Tuckerman, C. J. Mundy, S. Balasubramanian and M. L. Klein, J. Chem. Phys. 1065615 (1997).

[26] G. Marechal, J. P. Ryckaert and A. Bellemans, Mol. Phys. 6133 (1987).

[27] R. Edberg, D. J. Evans and G. P. Morriss, J. Chem. Phys. 875700 (1987).

[28] G. Marechal, J. P. Ryckaert and A. Bellemans, Mol. Phys. 6133 (1987).

[29] K. P. Travis, P. J. Daivis and D. J. Evans, J. Chem. Phys. 1031109 (1995).

[30] H. Goldstein, Classical Mechanics $2^{\text {nd }}$ ed. (AddisonWesley, London, 1980).

[31] S. Melchionna, Phys. Rev. E 616165 (2000).

[32] P. J. Morrison, Rev. Mod. Phys. 70467 (1998).

[33] A. R. Bishop, J. Phys. C 42241 (1971).

[34] J. P. Ryckaert and G. Ciccotti, Mol. Phys. 581125 (1986). 